Global Journal of Pure and Applied Mathematics.

ISSN 0973-1768 Volume 10, Number 3 (2014), pp. 443-449

(C) Research India Publications

https://dx.doi.org/10.37622/GJPAM/10.3.2014.443-449

\title{
A Novel Approach: Soft Topology
}

\author{
${ }^{*}$ V. Srinivasa Kumar and ${ }^{* *}$ B.V.S.T. Sai \\ Contact Author: * Dr. V. Srinivasa kumar, Assistant Professor, Department of \\ Mathematics, JNTUH College of Engineering, JNTU, Kukatpally, Hyderabad- \\ 500085, Telangana State, India. \\ ${ }^{* *}$ B.V.S.T. Sai, Research Scholar, Dept. of Mathematics, Andhra University, \\ Visakhapatnam, A.P, India.
}

\begin{abstract}
In this paper, we construct a soft topology on the universe set $X$ by assigning a topological structure to $X$ and investigate some interesting results in this context.
\end{abstract}

AMS Subject Classification: 54A10, 54A20, 54C08

Key Words: Soft set, Soft topology, Soft open set, Soft closed set, Soft closure, $s$-open set, $s$-closed set, Soft compactness, soft connectedness.

\section{Introduction}

In 1999, Molodtsov [7] introduced the soft set theory as a general mathematical tool for dealing with uncertainty or vagueness. There are many theories like Fuzzy set theory and Rough set theory to tackle the problem of imperfect knowledge. Soft set theory is still a better approach to deal with problems of uncertainty. Molodtsov recognized the importance of the role of parameters and introduced the theory of Soft sets. He has shown several applications of this theory in many fields like economics, engineering, medical sciences, etc. Later, this theory became a very good source of research for many mathematicians and computer scientists of recent years because of its wide range of applicability. The development in the fields of soft set theory and its application has been taking place in a rapid pace.

The notion of topological space for soft sets was first formulated by Shabir and $\mathrm{Naz}$ [10]. Later, many authors ([2] to [5]) have studied various properties of soft topological spaces. There are mainly two concepts in the theory of soft topology. One notion is to define a topology on the universe set and the second one is to make each 
soft set as a topological space. Mahanta and Das [6] introduced semi-soft open sets, semi-soft closed sets, soft semi-continuity and related concepts by following the first notion. Cagman, Karatas and Enginoglu [1] introduced the second notion and defined soft open sets, soft neighborhood, soft closure and related concepts.

In this present work, we introduce a soft topology on the universe set $X$ and some soft topological concepts like soft open sets, soft closed sets, soft closure, soft compactness and soft connectedness in a different approach by assigning a topological structure to $X$.

\section{Preliminaries}

In this section, we present some basic definitions which are needed in further study of this paper. Let $U$ be an initial universe set and $E_{U}$ (or simply $E$ ) be a collection of all possible parameters with respect to $U$, where parameters are the characteristics or properties of objects in $U$. Let $P(U)$ be the collection of all subsets of $U$.

1.1 Definition: A pair $(F, A)$ is called a soft set over $U$, if $A \subset E$ and $F: A \rightarrow P(U)$ . We write $F_{A}$ for $(F, A)$.

1.2 Definition: Let $F_{A}$ and $G_{B}$ be soft sets over a common universe set $U$ and $A, B \subset E$. Then we say that

(a) $F_{A}$ is a soft subset of $G_{B}$, denoted by $F_{A} \subset G_{B}$, if (i) $A \subset B$ and (ii) $F(e) \subset G(e) \forall e \in A$.

(b) $F_{A}$ equals $G_{B}$, denoted by $F_{A}=G_{B}$, if $F_{A} \subset G_{B}$ and $G_{B} \subset F_{A}$.

1.3 Definition: A soft set $F_{A}$ over $U$ is called a null soft set, denoted by $\Phi$, if $e \in A$, $F(e)=\phi$.

1.4 Definition: A soft set $F_{A}$ over $U$ is called an absolute soft set, denoted by $A$, if $e \in A, F(e)=U$.

1.5 Definition: The union of two soft sets $F_{A}$ and $G_{B}$ over a common universe $U$ is the soft set $H_{C}$, where $C=A \bigcup B$, and for all $e \in C$,

$$
H(e)=\left\{\begin{array}{l}
F(e) \text { if } e \in A-B \\
G(e) \text { if } e \in B-A \\
F(e) \cup G(e) \text { if } e \in A \cap B
\end{array}\right.
$$

We write $F_{A} \cup G_{B}=H_{C}$.

1.6 Definition: The intersection of two soft sets $F_{A}$ and $G_{B}$ over a common universe $U$ is the soft set $H_{C}$, where $C=A \cap B$, and for all $e \in C, H(e)=F(e) \bigcap G(e)$. We write $F_{A} \cap G_{B}=H_{C}$.

1.7 Definition: For a soft set $F_{A}$ over $U$, the relative complement of $F_{A}$ is denoted by $F_{A}^{c}$ and is defined by $F_{A}^{c}=F_{A}^{1}$, where $F^{1}: A \rightarrow P(U)$ is a mapping given by 
$F^{1}(e)=U-F(e)$ for all $e \in A$.

\section{Soft Topology and Related Concepts}

In this section, we present the definition of soft topology on a universe set and some related concepts. Henceforth, let $X$ be an initial universe set and $E$ be the fixed nonempty set of parameters with respect to $X$ unless otherwise mentioned.

2.1 Definition: A collection $T$ of subsets of $X$ is said to be a topology on $X$ if it satisfies the following properties.

(i) $\phi \in T$

(ii) $X \in T$

(iii)if $\left\{G_{\alpha}: \alpha \in \Delta\right\}$ is any collection of sets in $T$, then $\bigcup_{\alpha \in \Delta} G_{\alpha} \in T$

(iv) the intersection of any finite collection of sets in $T$ is a set in $T$.

The pair $(X, T)$ is called a topological space.

2.2 Definition: Let $\tau$ be the collection of soft sets over $X$. Then $\tau$ is called a soft topology on $X$, if $\tau$ satisfies the following properties.

(i) $\Phi, X$ belong to $\tau$.

(ii) The union of any number of soft sets in $\tau$ belongs to $\tau$.

(iii) The intersection of any two soft sets in $\tau$ belongs to $\tau$.

The triplet $(X, \tau, E)$ is called a soft topological space over $X$.

2.3 Definition: Let $F_{E}$ be a soft set over $X$ and $x \in X$. We say that $x \in F_{E}$ if $x \in F(e) \forall e \in E$. We say that $x \notin F_{E}$ if $x \notin F(e)$ for some $e \in E$.

2.4 Definition: Let $(X, \tau, E)$ be a soft topological space over $X$. Then

(i) The members of $\tau$ are called soft open sets in $(X, \tau, E)$.

(ii) A soft set $F_{E}$ over $X$ is said to be a soft closed set in $(X, \tau, E)$, if $F_{E}^{c} \in \tau$.

(iii) A soft set $F_{E}$ is said to be a soft neighborhood of a point $x \in X$ if $x \in F_{E}$ and $F_{E}$ is soft open in $(X, \tau, E)$.

(iv) The soft interior of a soft set $F_{E}$ is the union of all soft open subsets of $F_{E}$. The soft interior of $F_{E}$ is denoted by $\operatorname{Int}_{s}\left(F_{E}\right)$.

(v) The soft closure of $F_{E}$ is the intersection of all soft closed super sets of $F_{E}$. The soft closure of $F_{E}$ is denoted by $\mathrm{cl}_{s}\left(F_{E}\right)$.

(vi) A point $x \in X$ is said to be a soft limit point of a soft set $F_{E}$ if every soft neighborhood of $x$ contains a point of $F_{E}$ different from $x$.

\section{A New Approach : Soft Topological Concepts}


In this section, we adapt a new approach and introduce soft topological concepts in a different way by assigning a topological structure to the universe set $X$.

3.1 Definition: Let $(X, T)$ be a topological space. We say that a soft set $F_{E}$ is $s-$ open if $F(e) \in T$ for all $e \in E$. We denote the collection of all $s$-open sets with the symbol $\delta_{T}$.

3.2 Proposition: $\delta_{T}$ forms a soft topology over $X$.

Proof: Since $T$ is a topology on $X, \phi \in T$ and $X \in T$. Hence $\Phi \in \delta_{T}$ and $X \in \delta_{T}$ Let $\left\{\left(F_{\alpha}\right)_{E}: \alpha \in \Delta\right\}$ be any collection of $s$-open sets in $\delta_{T}$.

Then $\left(F_{\alpha}\right)_{E}(e) \in T$ for all $e \in E$ and for every $\alpha \in \Delta$

$\Rightarrow \bigcup_{\alpha \in \Delta}\left(F_{\alpha}\right)_{E}(e) \in T$ for all $e \in E$

$\Rightarrow \bigcup_{\alpha \in \Delta}\left(F_{\alpha}\right)_{E} \in \delta_{T}$.

Let $F_{E} \in \delta_{T}$ and $G_{E} \in \delta_{T}$. Then $F(e) \in T$ and $G(e) \in T$ for all $e \in E$

$\Rightarrow \quad F(e) \cap G(e) \in T$ for all $e \in E$

$\Rightarrow \quad F_{E} \cap G_{E} \in \delta_{T}$.

This shows that $\delta_{T}$ is a soft topology over $X$ and hence $\left(X, \delta_{T}, E\right)$ forms a soft topological space.

3.3 Definition: Let $(X, T)$ be a topological space. We say that a soft set $F_{E}$ is $s-$ closed if $(F(e))^{c}=X-F(e) \in T$ for all $e \in E$.

3.4 Proposition: In a soft topological space $\left(X, \delta_{T}, E\right)$,

(a) $F_{E}$ is $s$-open $\Leftrightarrow F_{E}$ is soft open over $X$.

(b) $F_{E}$ is $s$-closed $\Leftrightarrow F_{E}$ is soft closed over $X$.

(c) $F_{E}$ is $s$-open $\Leftrightarrow F_{E}^{c}$ is $s$-closed.

3.5 Example: Let $T=\{\phi, X\}$. Then $T$ is the indiscrete topology on $X$. Fix $e_{0} \in E$. If $F: E \rightarrow P(X)$ is defined by

$$
F(e)=\left\{\begin{array}{lll}
\phi & \text { if } & e=e_{0} \\
X & \text { if } & e \neq e_{0}
\end{array}\right.
$$

then this soft set $F_{E}$ is $s$-open and $F_{E} \in \delta_{T}$. From this example, it is clear that $\delta_{T}$ is not simply soft indiscrete eventhough $T$ is indiscrete.

3.6 Example: If $T=P(X)$, the discrete topology on $X$ then the soft topology $\delta_{T}$ corresponding to $T$ becomes the collection of all soft subsets over $X$. This soft topology $\delta_{T}$ is called the soft discrete topology over $X$. 
3.7 Definition: The soft closure of a soft set $F_{E}$ in $\left(X, \delta_{T}, E\right)$ is the intersection of all $s$-closed super sets of $F_{E}$. The soft closure of $F_{E}$ is the smallest $s$-closed set containing $F_{E}$.

3.8 Definition: The soft interior of a soft set $F_{E}$ in $\left(X, \delta_{T}, E\right)$ is the union of all $s-$ open subsets of $F_{E}$. The soft interior of $F_{E}$ is the largest $s$-open set contained in $F_{E}$

\section{Soft Compactness and Soft Connectedness}

In this section, we present a few interesting results on soft compactness and soft connectedness.

4.1 Definition: A soft topological space $(X, \tau, E)$ is said to be soft compact if every soft open cover of $X$ contains a finite sub cover.

4.2 Proposition: $(X, T)$ is compact $\Leftrightarrow\left(X, \delta_{T}, E\right)$ is soft compact.

Proof: Suppose that $(X, T)$ is compact. Let $\left\{\left(F_{\alpha}\right)_{E}: \alpha \in \Delta\right\}$ be a collection of $s-$ open sets over $X$ such that $X=\bigcup_{\alpha \in \Delta}\left(F_{\alpha}\right)_{E}$

$\Rightarrow X=\bigcup_{\alpha \in \Delta} F_{\alpha}(e)$ for any $e \in E$

Thus $\left\{F_{\alpha}(e): \alpha \in \Delta\right\}$ is an open cover of $X$. Since $(X, T)$ is compact, there exist finitely many indices $\alpha_{1}, \alpha_{2}, \alpha_{3}, \ldots \alpha_{n}$ in $\Delta$ such that $X=F_{\alpha_{1}}(e) \cup F_{\alpha_{2}}(e) \cup \ldots \cup F_{\alpha_{n}}(e)$ for every $e \in E$.

$\Rightarrow X=\left(F_{\alpha_{1}}\right)_{E} \cup\left(F_{\alpha_{2}}\right)_{E} \cup \ldots \cup\left(F_{\alpha_{n}}\right)_{E}$

Thus every soft open cover of $X$ has a finite subcover.

Hence $\left(X, \delta_{T}, E\right)$ is soft compact. Conversely, suppose that $\left(X, \delta_{T}, E\right)$ is soft compact. Let $\left\{G_{\alpha}: \alpha \in \Delta\right\}$ be an open cover of $X$. Then $X=\bigcup_{\alpha \in \Delta} G_{\alpha}$.

Define $F_{\alpha}: E \rightarrow P(X)$ such that $F_{\alpha}(e)=G_{\alpha} \quad \forall e \in E$.

Then $X=\bigcup_{\alpha \in \Delta}\left(F_{\alpha}\right)_{E}$. Since $\left(X, \delta_{T}, E\right)$ is soft compact, there exist finitely many indices $\alpha_{1}, \alpha_{2}, \alpha_{3}, \ldots \alpha_{n}$ in $\Delta$ such that $X=\left(F_{\alpha_{1}}\right)_{E} \cup\left(F_{\alpha_{2}}\right)_{E} \cup \ldots \cup\left(F_{\alpha_{n}}\right)_{E}$

$\Rightarrow X=G_{\alpha_{1}} \cup G_{\alpha_{2}} \cup \ldots \cup G_{\alpha_{n}}$

Thus every open cover contains a finite sub cover. Hence $(X, T)$ is compact.

4.3 Definition: A soft topological space $(X, \tau, E)$ is said to be soft disconnected if there exist two non null soft open sets $F_{E}$ and $G_{E}$ such that $X=F_{E} \cup G_{E}$ and $F_{E} \cap G_{E}=\Phi$. A soft topological space $(X, \tau, E)$ is said to be soft connected if it is not soft disconnected. 
4.4 Proposition: $(X, T)$ is disconnected $\Rightarrow\left(X, \delta_{T}, E\right)$ is soft disconnected.

Proof: Suppose that $(X, T)$ is disconnected. Then there exist two non-empty open sets $V$ and $W$ such that $X=V \bigcup W$ and $V \cap W=\phi$.

Define $F: E \rightarrow P(X)$ and $G: E \rightarrow P(X)$ such that $F(e)=V$ and $G(e)=W$ for all $e \in E$. Then $F_{E}$ and $G_{E}$ are two non null soft open sets in $\left(X, \delta_{T}, E\right)$ such that $X=F_{E} \cup G_{E}$ and $F_{E} \cap G_{E}=\Phi$. Hence $\left(X, \delta_{T}, E\right)$ is soft disconnected.

4.5 Proposition: $\left(X, \delta_{T}, E\right)$ is soft connected $\Rightarrow(X, T)$ is connected.

4.6 Remark: The converse of the above Proposition-(4.5) is not true. It is not necessary that $\left(X, \delta_{T}, E\right)$ is soft connected eventhough $(X, T)$ is connected. It can be seen from the following example.

4.7 Example: Let $X=\{a, b, c\}$ and $T=\{\phi, X,\{a\}\}$. Then $(X, T)$ is connected. Define $F: E \rightarrow P(X)$ and $G: E \rightarrow P(X)$ such that $F(e)=\left\{\begin{array}{lll}\phi & \text { if } & e=e_{0} \\ X & \text { if } & e \neq e_{0}\end{array}\right.$ and $G(e)=\left\{\begin{array}{lll}X & \text { if } & e=e_{0} \\ \phi & \text { if } & e \neq e_{0}\end{array}\right.$

Where $e_{0} \in E$ is a fixed parameter. Clearly $F_{E}$ and $G_{E}$ are two non null soft open sets in $\left(X, \delta_{T}, E\right)$ such that $X=F_{E} \cup G_{E}$ and $F_{E} \cap G_{E}=\Phi$.

Hence $\left(X, \delta_{T}, E\right)$ is soft disconnected.

\section{Soft $T_{1}$ and $T_{2}$ Spaces}

5.1 Definition: A $T_{1}$-space is a topological space in which, given any pair of distinct points, each has a neighborhood which does not contain other.

5.2 Definition: A topological space $(X, T)$ is said to be a $T_{2}$-space if for each pair of distinct points $x$ and $y$ in $X$ there exist two neighborhoods $V$ and $W$ of $x$ and $y$ respectively such that $V \cap W=\phi$.

5.3 Definition: We say that a soft set $F: E \rightarrow P(X)$ is a soft single point set if $F(e)=\{x\}$ for all $e \in E$ and for some $x \in X$.

5.4 Definition: We say that a soft topological space $(X, \tau, E)$ is a soft $T_{1}$-space if each soft single point set is soft closed in $(X, \tau, E)$.

5.5 Proposition: $(X, T)$ is $T_{1}-$ space $\Leftrightarrow\left(X, \delta_{T}, E\right)$ is a soft $T_{1}$ - space.

5.6 Definition: We say that a soft topological space $(X, \tau, E)$ is a soft $T_{2}$-space if 
each pair of distinct points $x$ and $y$ in $X$ there exist two soft neighborhoods $F_{E}$ and $G_{E}$ of $x$ and $y$ respectively such that $F_{E} \cap G_{E}=\Phi$.

5.7 Proposition: $(X, T)$ is $T_{2}$-space $\Leftrightarrow\left(X, \delta_{T}, E\right)$ is a soft $T_{2}$-space.

\section{References}

[1] Cagman. N., Karatas. S., and Enginoglu. S., Soft Topology, Comp. Math. Appl., Vol-62, pp. 351-358, 2011.

[2] Chen. B., Soft Semi-open Sets and related properties in Soft Topological Spaces, Appl. Math. Inf. Sci., Vol-7, No-1, pp. 287-294, 2013.

[3] Hussain. S., and Ahmed. B., Some Properties of Soft Topological Spaces, Comp. Math. Appl., Vol-62, pp. 4058-4067.

[4] Keun Min. W., A Note on Soft Topological Spaces, Comp. Math. Appl., Vol62, pp. 3524-3528, 2011.

[5] Li. F., Notes on the Soft Operations, Arpn Journal of System and Software, Vol-66, pp. 205-208, 2011.

[6] Mahanta. J., and Das. P. K., On Soft Topological Space Via Semi-open and semi-closed Soft Sets, Math. GN, Vol-14, pp. 1-8, 2012.

[7] Molodtsov. D., Soft Set Theory-First Results, Comp. Math. Appl., Vol-37, pp. 19-31, 1999.

[8] Ramabhadra Sarma, I., and V. Srinivasa kumar On Semi-Open Sets and Semi-Continuity, Journal of Advanced Studies in Topology, Vol. 3, No. 3, pp. 06-102, 2012.

[9] Sai. B.V.S.T., and Srinivasakumar. V., On Soft Semi-Open Sets and Soft Semi-Topology, International Journal of Mathematical Archive, Vol. 4, No. 3, pp. 114-17, 2013.

[10] Shabir. M., and Naz. M., On Soft Topological Spaces, Comp. Math. Appl., Vol-61, pp. 1786-1799, 2011.

[11] Simmons. G.F., Introduction to Topology and Modern Analysis, TATA McGraw-Hill, 1963. 